\title{
Vibration technology research achievements of the Mekhanobr scientific school and their practical implementation
}

\author{
Leonid Vaisberg \\ Mekhanobr-Tekhnika REC, St. Petersburg, Russia \\ E-mail: gornyi@mtspb.com \\ Received 17 May 2019; accepted 31 May 2019 \\ DOI https://doi.org/10.21595/vp.2019.20820
}

Check for updates

Copyright (C) 2019 Leonid Vaisberg. This is an open access article distributed under the Creative Commons Attribution License, which permits unrestricted use, distribution, and reproduction in any medium, provided the original work is properly cited.

\begin{abstract}
A brief overview of the achievements of the Mekhanobr Institute in the field of the theory and design of vibration processes and machines is given. The main theoretical works cover the discovery and development of the theory of self-synchronization of rotating bodies, the development of the theory of vibrational displacement, the creation of new analytical approaches to studying the effects of vibration on nonlinear systems and media - vibrational mechanics and vibrational rheology; the discovery and research into a number of nonlinear vibration effects, the development of the theory of vibrational classification. The main applied developments include the creation of a new class of highly efficient vibration machines (crushers, screens, separators, flotation machines, etc.), the development of respective design methods, the creation of a set of laboratory vibration equipment and a unique experimental base. The developments of recent years are considered in detail.
\end{abstract}

Keywords: vibration, nonlinear mechanics, vibrational mechanics, vibration machines, vibration technologies.

\section{Introduction}

The processing of natural and technogenic materials, and mineral processing in particular, is associated with the handling and processing of immense volumes of granular bodies and multicomponent media, such as highly concentrated slurries. In view of the special role of vibration in these processes, respective theoretical work and development of advanced vibration machines and technologies have been carried out at the Mekhanobr Institute since its establishment in 1916. This research and development work are now supported and promoted by Mekhanobr-Tekhnika Research and Engineering Corporation, founded in 1991.

The special effectiveness of vibration application in ore concentration is due to the fact that properties of materials, such as granular media and suspensions, tend to change under vibration. In particular, a granular medium may behave like a solid, a viscous liquid or a gas, depending on the vibration intensity. Under the action of vibration, the effective coefficients of dry friction are sharply reduced, including down to zero, and special additional forces emerge (referred to as vibration forces) that may be useful. Vibration enables changing and optimizing the process flow. In certain cases, it is possible to design environmentally friendly dry technologies and significantly reduce related energy costs.

The development work of Mekhanobr is carried out in active cooperation with a number of scientific and educational institutions from Russia and abroad. In particular, our Russian colleagues include the teams of the Institute of Problems of Mechanical Engineering of the Russian Academy of Sciences (IPME RAS), St. Petersburg, the Mechanical Engineering Research Institute of the Russian Academy of Sciences (Moscow), the St. Petersburg Mining, Polytechnic and Maritime Technical Universities, Moscow and St. Petersburg State Universities. As regards our foreign colleagues, close cooperation has been established with the teams from Great Britain, Germany, Denmark, Latvia, Lithuania, the Netherlands, Poland, the USA, Ukraine, the Czech Republic and Japan. The closest cooperation is maintained the IPME RAS, which has established 
and operates the joint Laboratory of Vibrational Mechanics.

The studies considered were conducted by an extensive team of scientists whose contribution is partially reflected in the references below. Employees of the Mekhanobr Institute are authors or co-authors in all monographs cited [1-24].

An important feature of Mekhanobr in the development of vibration technology is that it implements the full cycle, from the fundamental scientific research to machine design, technology development and industrial implementation.

\section{Major achievements in the theory of vibrational processes and devices}

The main theoretical achievements of Mekhanobr include:

- the development of the theory of vibrational displacement [1, 2];

- the discovery and elaboration of the theory of self-synchronization of rotating bodies (rotors), subsequently used as the basis for the development of a new class of vibration machines [3-6];

- the creation of new analytical approaches to studying the effects of vibration on nonlinear systems and media in the form of vibrational mechanics and vibrational rheology [7-14] and extensive practical application of these approaches, continuing the classical studies on the theory of nonlinear oscillations based on the methods developed by A. Poincaré, A.M. Lyapunov, N.M. Krylov, N.N. Bogolyubov, P.L. Kapitza, L.I. Mandelstam, N.D. Papaleksi and other researchers;

- detection, theoretical substantiation and analytical description of a number of nonlinear vibration effects and phenomena (vibroinjection, vibrational gradient segregation, abnormal segregation, vibrational maintenance and inhibition of rotation, abnormal behavior of solids and air bubbles in an oscillating fluid, vibrational rheological effects, including the formation of vibrational dynamic materials, stochastic resonance, etc.) [7-10];

- the development of the theory of vibrational classification and respective process and strength design methods for machines [15-22].

\section{Major applied developments}

The main applied achievements include: the creation of a new class of highly efficient vibratory crushers, mills, screens, feeders, conveyors, separators, and flotation machines, and development of respective design methods [9, 10, 15-24]; the creation of a set of laboratory equipment for studying concentration processes based on vibration effects; the creation of a respective experimental base, including a unique multipurpose vibration stand; participation in the creation of the six-volume encyclopedic handbook titled "Vibrations in Engineering" [25].

Over 1,500 copyright certificates and patents for inventions and utility models have been registered for the developments of Mekhanobr. The equipment designed by Mekhanobr-Tekhnika REC is supplied to many countries of the world and manufactured under licenses. A joint machinebuilding enterprise has been established with the People's Republic of China.

\section{On certain theoretical developments of recent years}

1. The development of a general analytical method for studying the effects of vibration on nonlinear dynamic systems has been continued for vibrational mechanics and vibrational rheology and their generalization, i.e. oscillatory strobodynamics. The scientific foundations of these approaches are presented in monographs $[9,10]$ and developed in $[26,27]$ and the works by I. I. Blekhman and V. S. Sorokin (2016) published in Procedia IUTAM 19 and Nonlinear Dynamics (Vol. 83, Issue 4), which also contain numerous examples of their practical application for solving relevant applied problems from various fields of knowledge.

2. The vibrational mechanics approach has been extended to include systems with random 
effects. With regard to vibration machines, the practical significance of this generalization is due to the fact that the properties and composition of the process load in these machines tend to vary randomly. The results of respective studies are presented in the works by I. I. Blekhman, E.B. Kremer and V.S. Sorokin published in Journal of Sound and Vibration (2018, vol. 437); Nonlinear Dynamics (2018, vol. 93, Issue 2); European Journal of Applied Mathematics (2018) and Journal of Mechanical Engineering Science. Part C (2019).

3. A series of studies on the problem of stochastic resonance have been carried out (see the references in the previous paragraph). The regularities of this phenomenon have been established and described for various nonlinear systems. A physical explanation and a simple mathematical description of the stochastic resonance phenomenon have been provided, based on the vibrational mechanics approach. It has been established that random effects are not a mandatory requirement for the occurrence of this phenomenon and that a simple harmonic effect is sufficient. In this regard, the very term "stochastic resonance" is viewed as misleading for the researchers. The practical significance of the research consists in the extension of the scope of applications for the resonance phenomenon in vibration technology and engineering. In electrical and radio engineering, however, its use remains very limited. This is largely due to the difficulties in controlling the mechanical properties of materials.

4. The equation of vibrational mechanics has been obtained, which describes the motion of a complex actual machine with several degrees of freedom. It essentially generalizes the classical equation. The equation is used in the analysis of resonances during the start-up and run-out of a vibration machine - see the works by I. I. Blekhman, and E. B. Kremer (2017) published in Journal of Machinery Manufacture and Reliability (Vol. 46, Issue 4), Journal of Sound and Vibrations (Vol. 405) and Procedia Engineering (Vol. 199).

5 . The idea of creating fundamentally new materials, the so-called dynamic materials and composites, has been put forward and developed $(2000)[9,10,28]$. Dynamic materials refer to the media with their material parameters (density, rigidity, dissipative characteristics, selfinduction, capacity, etc.) changing both in space and in time. Examples of the implementation of dynamic materials are given in [10]. Vibratory dynamic materials and composites are an important class in this regard (2007) $[9,10]$. Vibratory dynamic materials are materials, the parameters and properties of which with respect to relatively slow or static effects are significantly changed under the influence of vibration, i.e. relatively rapid impacts. Vibrational rheology lays down the theoretical basis for the creation of such materials.

6. The laws governing the unusual behavior of solid particles and air bubbles in a liquid under the action of vibration have been considered [9, 10, 29-31].

7. The possibilities of improving vibration machines with self-synchronizing inertial (unbalanced) vibration exciters with superresonance working oscillation frequencies have been considered. The improvements proposed allow reducing the installed motor power, energy consumption and dynamic loads in operation of the machines. Oscillations in the drives of vibration machines with inertial (unbalanced) oscillation exciters have also been theoretically and experimentally studied, in which the shafts of the electric motor and the exciter are coupled so as to enable relative displacement of the using an elastic coupling, V-belt drive, universal-joint shaft - see the works by N. P. Yaroshevich et. al. (Journal of Machinery Manufacture and Reliability, 2013, vol. 42, Issue 3 and Obogashchenie Rud (Mineral Processing J., 2017, Iss. 4).

8. The main sources of energy consumption in vibrational transportation and process machines, have been studied. The studies focus on the energy consumption in these machines that is required to ensure the vibration effect on the material being processed and to overcome the resistance forces in the drive. The power required to maintain the oscillatory motion in the steady state and the motor power required to start the machines are also considered. It is shown that starting power values may be practically reduced to zero using relatively simple motor start control systems. The comparison of the calculated data against the technical specifications of actual machines indicates that, for example, the installed power of a number of screens may be significantly reduced by reducing or eliminating the start-up consumption. The research results 
are published in Obogashchenie Rud (Mineral Processing Journal, 2019, Issue 1).

9. The phenomenon of vibrational injection of gases into liquids has been discovered and studied $[9,10,32]$. This phenomenon is observed when air or gas is intensively sucked in series of bubbles through holes in the lower part of a vibrating vessel filled with liquid. This effect is used in the mining industry and may also be applied to intensify industrial processes and in various vibration devices, for example, for dispensing and aeration (Patents RU 2263883 and RU 2278738). It has been shown that vibrational injection and the previously known other nonlinear phenomenon of the vibrating-jet effect may be considered jointly as special cases of a more general theory. These effects are not only useful but have previously also caused accidents in the chemical industry and might have been the cause of a number of aviation accidents when, due to vibration, fuel supply from the gas tanks was cut off due to vibrational locking of the holes. Ways to suppress these undesirable phenomena have been developed.

10. The wear of contacting nominally fixed machine parts operating under systematic vibration or shock effects (fretting wear) has been studied. The wear is due to micro- and sometimes macro-mobility of joints. At the level of simple physical models, formulas for the wear rate have been obtained and recommendations have been developed on the design and operation of the corresponding equipment. The relative shift (microslippage) of contacting bodies under highfrequency effects caused by shocks has also been studied. The amount of the microslippage has been determined that allows estimating the wear rate and the trouble-free service life for the parts. Expressions for the effective coefficients of dry friction have been obtained, indicating a significant (down to zero) reduction of these coefficients under shock loads. The models considered are quite universal, may be used to explain and describe certain technogenic seismic phenomena and are applicable to the problem of increasing the efficiency of oil-bearing formations using vibration effects - see Obogashchenie Rud (Mineral Processing Journal) (2011, Issue 6; 2016, Issue 6) and Doklady Physics (2017, Vol. 62, Issue 5).

11. A series of studies have been carried out on the phenomenon of vibrational segregation of granular bodies, which underlies the processes of material separation $[9,10,33]$.

a) The possibility of two types of the process has been established and studied. In the first case, segregation occurs in the direction of reduction of the potential energy of the system (normal segregation). In the second case, vice versa, the process occurs in the direction of increasing the potential energy, in particular, when large heavy particles float with fine and light particles (the Brazil nut effect). The latter is implemented in regular screens. It also forms the basis of the newly developed screenless wedge wire classifiers (Patent RU 2407600).

b) The effect referred to as vibrational diffusion (gradient) segregation has been discovered and studied [33]. It is observed when, under sufficiently intense vibration applied to a granular mixture, the particles of each specific size (fraction) slowly (as compared with the vibration rate) move in the direction of their lowest concentration, that is, in the direction opposite to the direction of the concentration gradient for this fraction. The motion of relatively fine particles in a vibrating granular medium is similar to the processes of matter propagation during diffusion and heat propagation. A significant result of these studies indicates that, with sufficiently intense vibration (up to $10 \mathrm{~g}$ ), the fines fraction is discharged more efficiently through inclined and, in particular, vertical screening surfaces of the tray than through the traditional bottom surfaces. Based on this effect, new high-performance screens have been developed.

12. A cycle of studies have been carried out to develop measures to prevent or reduce the risk of accidents at industrial facilities, structures and vehicles caused by non-linear oscillatory phenomena (see [9, 10,32], as well as paragraphs 4, 9, 10 above and the references cited therein). The phenomena of vibrational braking and vibrational maintenance of rotation, the phenomenon that occurs when several unbalanced machines are installed on a single foundation, the phenomenon of vibrational injection of gas into liquid, the associated phenomenon of liquid locks in holes of vibrating vessels, and the phenomenon of relative shifts of contacting bodies due to weak vibration or shock effects have been studied. In particular, the mechanisms ensuring a significant reduction in the effective coefficient of dry friction under weak shock and vibration 
effects have been investigated. The magnitude of the resulting displacement of the bodies has been estimated. The following groups of accidents have been explained and described on this basis: the influence of technogenic factors on seismic phenomena; the impact of granular materials and loose cargo on the accident rate for vessels carrying such cargo under rolling conditions; the wear rate of the contacting surfaces of nominally motionless machine parts; the manifestation mechanism of the Sommerfeld effect for irregular travel paths.

13. Methods have been developed for damping harmful vibrations, including through the use of harmful vibrations to generate energy (Patents RU 2678932; RU 2637156). The latter method is based on the use of the phenomenon of vibrational maintenance of rotation and the phenomenon of vibrational locking of rotation of an unbalanced rotor [9].

14. The phenomenon of oscillation-induced suspension of a heavy body vibrating in a liquid near a solid wall has been investigated and explained. Expressions have been obtained for the suspension force and height [34]. The results may be used in the theory of suspension flows, the theory of vibration pumps, etc. The increase in buoyancy of a vibrating body near the interface has been noted and investigated. The difference in the physical mechanisms of suspension for heavy bodies in a liquid and in a granular medium has been considered.

15. The task has been set to study vibrational transportation of solid and granular bodies along non-translationally vibrating surfaces and respective studies have been initiated. The specific features of the emerging motion suggest that the results of these studies will enable developing new vibration devices for processing natural and technogenic materials. These results must also be taken into account when analyzing the behavior of loose cargo on sea vessels under rolling conditions. This and a number of other new developments are described in the reports prepared by employees of Mekhanobr-Tekhnika REC for Vibroengineering 2019.

\section{Practical applications of the research results}

On the basis of the research conducted at Mekhanobr-Tekhnika REC, a new class of highly efficient vibration machines (crushers, mills, screens, feeders, conveyors, separators, flotation machines) was created and widely introduced in the industry. In particular, a range of industrial inertia cone crushers has been developed. The specific feature of their design is that the motion of the crushing head is ensured by an inertial vibration exciter instead of an eccentric. These machines provide improved disintegration rates for high-strength and extra strong materials, such as granite, ferroalloys, etc. Vibratory cone crushers with vertical reciprocating motion of the crushing head effectively disintegrate fragile materials, for example, corundum or glass-apatites with the minimal overgrinding of respective raw materials. Double-jaw vibratory crushers with high-frequency and low-frequency oscillations are indispensable for the reduction of waste reinforced concrete and hard rocks. Vibrating horizontal and vertical feeders ensure accurate metering of granular materials in crushing and separation process systems. Vibrating screens for grain-size classification of various types, developed and supplied by Mekhanobr-Tekhnika, have the screening surface areas of 0.5 to $20 \mathrm{~m}^{2}$ and, in terms of their efficiency and reliability, rate among the best in the world. A number of machine types are exported.

On the basis of the equipment designed, Mekhanobr-Tekhnika manufactures and supplies modular plants and process lines for the processing of mineral and technogenic raw materials, in particular, crushing and screening plants for the production of non-metallic building materials and recycling facilities for construction waste, waste paper, electronic and electrical scrap and cable, waste of hard alloys, rubber and plastic waste, lines for the manufacture and final treatment of powder materials for additive manufacturing and powder metallurgy.

A wide range of laboratory vibration equipment has been developed to study the processing of materials. This equipment is in demand for equipping research laboratories of mining plants and university teaching laboratories and is acquired for use as the main production equipment at smallscale production facilities. The complete set of laboratory equipment is available at the non-profit research and educational center, which is part of Mekhanobr-Tekhnika REC. The universal 
vibration stand designed by Mekhanobr holds a special place in the range of its test equipment. Through simple reconfigurations, stand enables obtaining oscillations with various types and orientations of trajectories in space. This makes it indispensable when studying and optimizing industrial processes, as well as when researching the effects of vibration on the properties of complex media, materials and structures. The stand may also be used for educational purposes to demonstrate peculiar vibration effects. A number of new nonlinear effects have already been discovered and investigated with the use of the stand, in particular, the phenomenon of vibration injection of gas into liquid. The unique capabilities of the stand are due to the phenomenon of self-synchronization of mechanical vibration exciters.

\section{Acknowledgements}

The study was carried out at the expense of the grant of the Russian Science Foundation No. 17-79-30056 (the project of the Mekhanobr-Tekhnika REC).

\section{References}

[1] Blekhman I. I., Dzhanelidze Yu G. Vibrational Movement. Nauka, Moscow, 1964, p. 410.

[2] Nagaev R. F. Periodic Regimes of Vibrational Displacement. Nauka, Moscow, 1978, p. 160.

[3] Blekhman I. I. Synchronization of Dynamic Systems. Nauka, Moscow, 1971, p. 895.

[4] Blekhman I. I. Synchronization in Science and Technology. ASME Press, New York, 1988, p. 255.

[5] Nagaev R. F., Guzev V. V. Self-Synchronization Inertial Vibration Exciters. Mashinostroenie (Mech. Engineering), Leningrad, 1990, p. 178.

[6] Nagaev R. F. Quasi-Conservative Synchronizing Systems. Nauka, St. Petersburg, 1996, p. 252.

[7] Blekhman I. I. Vibrational Mechanics. World Scientific, 2000, p. 510.

[8] Selected Topics in Vibrational Mechanics. Series A, Vol. 11. World Scientific, New Jersey. 2004.

[9] Blekhman I. I. Theory of Vibrational Processes and Devices: Vibrational Mechanics and Vibrational Technology. Ruda I Metalli (Ore and Metals), St. Petersburg, 2013, p. 640.

[10] Blekhman I. I. Vibration Mechanics and Vibrational Rheology (Theory and Applications). Fizmatlit, Moscow, 2018, p. 752.

[11] Nagaev R. F., Khodzhaev Sh K. Oscillations of Mechanical Systems with Periodic Structure. FAN Publishing House, Tashkent, 1973, p. 272.

[12] Nagaev R. F. Mechanical Processes with Repeated Attenuated Impacts. World sc., Singapore, 1999.

[13] Skubov Yu D., Khodzhaev Sh K. Asymptotic Qualitative Methods in the Theory of Synchronous Electric Machines. Publishing House of Saint-Petersburg State Institution of Technology, 1999, p. 155.

[14] Skubov D., Khodzhaev Sh K. Non-Linear Electromechanics. Springer, Berlin, 2008, p. 400.

[15] Olevsky V. A. Designing and Calculation of Screens. Metallurgizdat, Moscow, 1955, p. 124.

[16] Kizevalter B. V. Theoretical Basis of Gravity Beneficiation Processes. Nedra, Moscow, 1979, p. 295.

[17] Vaisberg L. A. Design and Calculation of Vibrating Screens. Nedra, Moscow, 1986, p. 144.

[18] Vaisberg L. A., Rubisov D. G. Vibration Screening of Bulk Materials. Process Modeling and Technological Calculations for Screens. Mekhanobr, St. Petersburg, 1994, p. 47.

[19] Vaisberg L. A., Zarogatsky L. P., Turkin Ya V. Vibratory Crushers. Bases for Design, Engineering and Technological Applications. VSEGEI Publishing House, St. Petersburg, 2004, p. 306.

[20] Ivanov N. A. The inertial Cone Crusher. Ore and Metals, St. Petersburg, 2012, p. 128.

[21] Anakhin V. D., Pliss D. A., Monakhov V. N. Vibration Separators. Nedra, Moscow, 1991, p. 157.

[22] Anakhin V. D., Pliss D. A. On the Theory of Vibration Separators. Novosibirsk, 1992, p. 125.

[23] Vaisberg L. A., Kartavy A. N., Korovnikov A. N. Screening Surfaces of Screens. Designs, Materials, Application Experience. VSEGEI Publishing House, St. Petersburg, 2005, p. 252.

[24] Arsentiev V. A., Vaisberg L. A., Zarogatsky L. P., Shuloyakov A. D. The Production of Cuboid Crushed Stone and Building Sand Using Vibrating Crushers. 2 ed., VSEGEI Publishing House, St. Petersburg, 2008, p. 112.

[25] Vibrations in Engineering: Handbook in 6 volumes. Mashinostroenie (Mechanical Engineering), Moscow, p. 1978-1981, (in Russian).

[26] Sorokin V. S. On the unlimited gain of a nonlinear parametric amplifier. Mechanics Research Communications, Vol. 62, 2014, p. 111-116. 
[27] Kremer E. Slow motions in systems with fast modulated excitation. Journal of Sound and Vibration, Vol. 383, 2016, p. 295-308.

[28] Lurie K. A. An Introduction to the Mathematical Theory of Dynamic Mathematical. Springer New-York, 2007, p. 181.

[29] Blekhman I. I., Blekhman L. I., Vaisberg L. A., Vasilkov V. B., Yakimova K. S. "Anomalous" phenomena in fluid under the action of vibration. Doklady Physics, Vol. 53, Issue 10, 2008, p. 520-524.

[30] Blekhman I. I., Blekhman L. I., Sorokin V. S., Vasilkov V. B., Yakimova K. S. Surface and volumetric effects in a fluid subjected to high-frequency vibration. Proceedings of the Institution of Mechanical Engineers, Part C: Journal of Mechanical Engineering Science, Vol. 226, Issue 8, 2012, p. 2028-2043.

[31] Demidov I. V., Sorokin V. S. Motions of deformable inclusions inahorizontally oscillating vessel with a compressible fluid. Journal of Sound and Vibration, Vol. 383, 2016, p. 324-338.

[32] Blekhman I. I., Blekhman L. I., Vaisberg L. A., Vasilkov V. B., Yakimova K. S. Nonlinear effects observed in the flow of a fluid out of vibrating vessels. Doklady Physics, Vol. 48, Issue 7, 2003, p. 355-358.

[33] Blekhman I. I., Blekhman L. I., Vaisberg L. A., Vasilkov V. B., Yakimova K. S. On vibrational diffusion segregation in granular media. Doklady Physics, Vol. 61, Issue 1, 2016, p. 8-10.

[34] Blekhman L. I. Oscillation-induced suspension of solids in fluid and loose media. PNRPU Mechanics Bulletens, Vol. 2, 2013, p. 52-83, (in Russian). 